\title{
Landtagswahlen in Rheinland-Pfalz 1951 bis 2001: starke bundespolitische Einflüsse, große Bedeutung der Parteibindung
}

\author{
Jürgen Maier
}

Rheinland-Pfalz hat in den 1990er Jahren eine politische Metamorphose durchlebt: Aus dem einstigen „christdemokratischen Stammland“" in dem die CDU mehr als vier Jahrzehnte - zwischen 1971 bis 1983 sogar ohne den langjährigen Koalitionspartner FDP² die Regierungsgeschäfte führte und seit Gründung des Landes immer den Ministerpräsidenten stellte, ist seit 1991 ein sozialdemokratisch regiertes Bundesland geworden (vgl. Tabelle 1). Die Erosion der „Hegemonialstellung“3 der CDU zeichnete sich schon Mitte der 1970er Jahre ab, denn mit Ausnahme des Jahres 1983 - erstmals in der Geschichte der Bundesrepublik wurde hier eine Landtagswahl gleichzeitig mit einer Bundestagswahl abgehalten ${ }^{4}$ - musste sie seit 1975 einen bis heute andauernden, stetigen Verlust an Wählerstimmen hinnehmen, der in den Jahren 1987 und 1991 besonders deutlich ausfiel. ${ }^{5}$ Dieser Trend ist auch bei Bundestagswahlen zu erkennen: Bis 1983 (mit Ausnahme von 1972) pendelten die Wahlergebnisse der CDU stets um die 50-Prozent-Marke und bewegten sich damit deutlich über den in anderen Bundesländern erzielten Resultaten. 6 Seit 1987 verlieren die Christdemokraten jedoch kontinuierlich an Stimmen; bei der Bundestagswahl 2005 mussten sie sogar das schlechteste je in Rheinland-Pfalz erzielte Ergebnis hinnehmen.

Begünstigt durch den Niedergang der CDU konnte die SPD bei der Landtagswahl 1991 erstmals stärkste Partei werden, nachdem sie zuvor Wahlergebnisse erzielt hatte, die bis zu 15 Prozentpunkte unter dem CDU-Ergebnis lagen. Wenngleich Zweifel daran bestehen, dass die Umorientierung der Wähler von Dauer ist ${ }^{7}$, haben die Sozialdemokraten die Regierungsmacht seither nicht mehr abgegeben. ${ }^{8}$ Bei der Landtagswahl $2006^{9}$ konnten sie sich sogar - dank des knappen Scheiterns von Bündnis 90/Die Grünen an der Fünf-Pro-

1 Kai Arzheimer, 50 Jahre Wahlen in Rheinland-Pfalz, in: Ulrich Sarcinelli / Jürgen W. Falter / Gerd Mielke / Bodo Benzner (Hrsg.), Politische Kultur in Rheinland-Pfalz, Mainz 2000, S. 240.

2 Bereits bei den Landtagswahlen 1955 und 1959 gelang es der CDU, die absolute Mehrheit der Mandate auf sich zu vereinigen. Dennoch bildeten sie eine Koalition mit der FDP.

3 Vgl. Gerd Mielke / Ulrich Eith, Die Landtagswahl 1991: Erdrutsch oder Ausrutscher? Parteienkonkurrenz und Wählerbindungen in Rheinland-Pfalz in den 80er und 90er Jahren, in Ulrich Sarcinelli / Jürgen W. Falter / Gerd Mielke / Bodo Benzner (Hrsg.), a.a.O., S. 255 ff.

4 Vgl. Peter Haungs / Eckhard Jesse, Die rheinland-pfälzische Landtagswahl vom 6. März 1983. Die erste „Doppelwahl“ in der Geschichte der Bundesrepublik, in: ZParl, 14. Jg. (1983), H. 4, S. $517 \mathrm{ff}$.

5 Kai Arzheimer / Cornelia Weins: Zerfallen die sozialstrukturellen Bindungen an die Union - zum Beispiel in Rheinland-Pfalz?, in: ZParl, 28. Jg. (1997), H. 2, S. 205.

6 Vgl. Kai Arzheimer, a.a.O.

7 Vgl. Gerd Mielke / Ulich Eith, a.a.O., S. 276 f.

8 Allerdings war die SPD bereits während der ersten Wahlperiode an der Landesregierung beteiligt. 1947 bildeten die Sozialdemokraten eine Allparteienkoalition mit der CDU, der FDP und der KPD. Zwischen 1948 und 1951 (ausgenommen einer zweimonatigen Phase im Jahr 1949, in der die CDU eine Minderheitsregierung bildete) schloss sich die SPD mit der CDU zu einer Großen Koalition zusammen.

9 Vgl. Heiko Gothe, Die rheinland-pfälzische Landtagswahl vom 26. März 2006: „König Kurt“ erringt die absolute Mehrheit, in: ZParl, 38. Jg. (2007), H. 1, S. 34 ff. 


\begin{tabular}{|c|c|c|c|c|c|}
\hline \multicolumn{6}{|c|}{$\begin{array}{l}\text { Tabelle 1: Landtags- und Bundestagswablergebnisse in Rheinland-Pfalz, } 1947 \text { bis } 2006 \\
\text { (Angaben in Prozent) }\end{array}$} \\
\hline Jahr & $\mathrm{CDU}$ & SPD & FDP & B90/Grüne & Sonstige \\
\hline \multicolumn{6}{|c|}{ Landtagswahlen } \\
\hline 1947 & 47,2 & 34,3 & 9,8 & - & 8,7 \\
\hline 1951 & 39,2 & 34,0 & 16,7 & - & 10,1 \\
\hline 1955 & 46,8 & 31,7 & 12,7 & - & 8,8 \\
\hline 1959 & 48,4 & 34,9 & 9,7 & - & 7,0 \\
\hline 1963 & 44,4 & 40,7 & 10,1 & - & 4,8 \\
\hline 1967 & 46,7 & 36,8 & 8,3 & - & 8,2 \\
\hline 1971 & 50,0 & 40,5 & 5,9 & - & 3,5 \\
\hline 1975 & 53,9 & 38,5 & 5,6 & - & 1,9 \\
\hline 1979 & 50,1 & 42,3 & 6,4 & - & 1,2 \\
\hline 1983 & 51,9 & 39,6 & 3,5 & 4,5 & 0,5 \\
\hline 1987 & 45,1 & 38,8 & 7,3 & 5,9 & 2,9 \\
\hline 1991 & 38,7 & 44,8 & 6,9 & 6,5 & 3,2 \\
\hline 1996 & 38,7 & 39,8 & 8,9 & 6,9 & 5,7 \\
\hline 2001 & 35,3 & 44,7 & 7,8 & 5,2 & 6,9 \\
\hline 2006 & 32,8 & 45,6 & 8,0 & 4,6 & 9,0 \\
\hline \multicolumn{6}{|c|}{ Bundestagswahlen } \\
\hline 1949 & 49,0 & 28,6 & 15,8 & - & 6,6 \\
\hline 1953 & 52,1 & 27,2 & 12,1 & - & 8,6 \\
\hline 1957 & 53,7 & 30,4 & 9,8 & - & 6,1 \\
\hline 1961 & 48,9 & 33,5 & 13,2 & - & 4,4 \\
\hline 1965 & 49,3 & 36,7 & 10,2 & - & 3,8 \\
\hline 1969 & 47,8 & 40,1 & 6,3 & - & 5,8 \\
\hline 1972 & 45,9 & 44,9 & 8,1 & - & 1,1 \\
\hline 1976 & 49,9 & 41,7 & 7,6 & - & 0,9 \\
\hline 1980 & 45,6 & 42,8 & 9,8 & 1,4 & 0,5 \\
\hline 1983 & 49,6 & 38,4 & 7,0 & 4,5 & 0,5 \\
\hline 1987 & 45,1 & 37,1 & 9,1 & 7,5 & 1,3 \\
\hline 1990 & 45,6 & 36,1 & 10,4 & 4,0 & 3,8 \\
\hline 1994 & 43,8 & 39,4 & 6,9 & 6,2 & 3,7 \\
\hline 1998 & 39,1 & 41,3 & 7,1 & 6,1 & 6,4 \\
\hline 2002 & 40,2 & 38,2 & 9,3 & 7,9 & 4,3 \\
\hline 2005 & 36,9 & 34,6 & 11,7 & 7,3 & 9,5 \\
\hline
\end{tabular}

zent-Hürde - des liberalen Koalitionspartners „entledigen“ und regieren erstmals in der Geschichte des Landes allein. Rheinland-Pfalz wurde damit endgültig zu einer neuen sozialdemokratischen Bastion. Im Gegensatz zur CDU zeigen sich bei der SPD jedoch keine besonders starken Parallelitäten zwischen Bundes- und Landtagswahlergebnissen: So sorgte in Rheinland-Pfalz „Genosse Trend“ zwischen 1949 und 1972 für bessere Wahlergebnisse von Bundestagswahl zu Bundestagswahl - eine Entwicklung, die auf Landesebene nicht zu erkennen ist. Seit Anfang der 1980er Jahre erzielte die SPD wieder schlechtere Resultate bei Bundestagswahlen und legte erst 1994 und 1998 (hier gelang es ihr zum bislang ersten 
Mal, die CDU als stärkste Kraft abzulösen) wieder zu. Im Unterschied zu den Landtagswahlen 2001 und 2006 konnte die SPD diesen Aufwärtstrend bei Bundestagswahlen jedoch nicht fortsetzen, sondern fiel auf das Niveau der 1960er Jahre zurück.

Als Ursache für den Bedeutungsverlust der CDU wird in erster Linie die Auflösung tradierter Bindungen der bis heute geographisch vor allem in der Eifel, entlang von Mosel und Saar, dem Westerwald sowie der Südpfalz konzentrierten katholischen Bevölkerungsmehrheit an die CDU angeführt: Einstige christdemokratische Hochburgen haben sich stark abgeschliffen; der SPD gelang es, hier aufzuschließen. Umgekehrt konnte die CDU ihre Stellung in bisherigen so genannten Diasporagebieten festigen, während die SPD dort, wo sie früher besonders gute Ergebnisse erzielte, an Bedeutung eingebüßt hat. ${ }^{10}$ Diese Entwicklung wird zum einen auf Modernisierungsprozesse wie etwa die wachsende Bedeutung des Dienstleistungssektors oder die fortschreitende Säkularisierung der Gesellschaft zurückgeführt. ${ }^{11}$ Eine Reihe von „Fehlleistungen“ der CDU in den 1980er und 1990er Jahren innerparteiliche Konflikte, die Abwahl von Ministerpräsident Bernhard Vogel als CDU-Vorsitzendem sowie die Schwäche der Bundes-CDU nach der ersten gesamtdeutschen Bundestagswahl - beschleunigte die Auflösung sozialstrukturell bedingter Parteiloyalitäten weiter. ${ }^{12}$ Zum anderen wird das Aufkommen der Grünen, das anders als in anderen Teilen Deutschlands in Rheinland-Pfalz nicht ausschließlich zu Lasten der SPD ging und die deshalb bei Landtagswahlen nie mehr als sieben Prozent der Wähler an sich binden konnten, als Ursache für die ungewöhnliche Veränderung der politischen Landschaft gesehen. Dies gilt vor allem im Vergleich zu anderen Bundesländern, in denen eher eine Ausdifferenzierung des Parteiensystems zu beobachten ist. ${ }^{13}$

Als Folge der Entkoppelung von Bürgern und Parteien rücken zunehmend kurzfristige politische Orientierungen in den Mittelpunkt, wenn es darum geht, das Stimmverhalten der Wähler zu erklären. Dies ist auch in Rheinland-Pfalz so. ${ }^{14}$ Neben den klassischen Kurzfristfaktoren - den Einstellungen gegenüber dem im Land zur Wahl stehenden politischen Personal sowie die Bewertung der Problemlösungskapazität der Landesparteien - stehen auch die bundespolitischen Konstellationen im Verdacht, das Landtagswahlverhalten zu beeinflussen. Dies ergibt jedenfalls die Durchsicht der in der Zeitschrift für Parlamentsfragen veröffentlichten Analysen der rheinland-pfälzischen Landtagswahlen. So wurde etwa die Landtagswahl 1975 als Plebiszit über eine mögliche Kanzlerkandidatur von Ministerpräsident Helmut Kohl eingeschätzt. ${ }^{15}$ Auch wiesen die im Wahlkampf diskutierten Themen mehrheitlich einen bundespolitischen Bezug auf. ${ }^{16} 1979$ sorgte der Machtkampf in der

10 Vgl. Kai Arzheimer / Cornelia Weins, a.a.O.; Gerd Mielke / Ulrich Eith, a.a.O., S. 263 ff.

11 Oscar W. Gabriel / Werner Jann / Rudolf Deinert, Rheinland-Pfalz, in: Jürgen Hartmann (Hrsg.), Handbuch der deutschen Bundesländer, 3. Auflage, Frankfurt am Main 1997, S. 453; Gerd Mielke / Ulrich Eith, a.a.O., S. 262.

12 Vgl. Kai Arzheimer, a.a.O., S. 250; Werner Billing, Die rheinland-pfälzische Landtagswahl vom 21. April 1991: Machtwechsel in Mainz nach 44 Jahren, in: ZParl, 22. Jg. (1991), H. 4, S. 598; Oscar W. Gabriel / Werner Jann / Rudolf Deinert, a.a.O., S. 454; Gerd Mielke / Ulrich Eith, a.a.O., S. 262.

13 Gerd Mielke / Ulrich Eith, a.a.O., S. 262.

14 Oscar W. Gabriel / Werner Jann / Rudolf Deinert, a.a.O., S. 454.

15 Rüdiger Andel, Die rheinland-pfälzische Landtagswahl vom 9. März 1975. Erster Test für einen Kanzlerkandidaten der Opposition?, in: ZParl, 6. Jg. (1975), H. 4, S. 466.

16 Ebenda. 
Bundes-CDU für Gesprächsstoff ${ }^{17}$, vier Jahre später dominierte der Zusammenbruch der sozial-liberalen Koalition in Bonn den Landtagswahlkampf. ${ }^{18}$ Die Landtagswahl 1991 wurde zur Abrechnung der Wähler mit dem Kurs der Bundesregierung bei der Finanzierung der deutschen Einheit. ${ }^{19} 1996$ standen wirtschaftspolitische Themen sowie eine mögliche Ablösung der Regierungskoalition im Bund bei der Wahl 1998 durch Rot-Grün und - ein Scheitern der FDP an der Sperrklausel vorausgesetzt - ein Testlauf dieser Variante in Rheinland-Pfalz auf der Agenda. ${ }^{20}$ Die Landtagswahl 2001 stand unter dem Eindruck der CDUParteispendenaffäre, die zwischenzeitlich auch die rheinland-pfälzische Union erreicht hatte. Auch Bündnis 90/Die Grünen hatten mit bundespolitischem Gegenwind zu kämpfen. ${ }^{21}$ Demgegenüber berichten die Wahlanalysen nur in zwei Fällen - 1971 und 1987 - von einem geringen Einfluss der Bundespolitik auf rheinland-pfälzische Landtagswahlen. ${ }^{22}$ Diese Einschätzung dürfte auch für die Landtagswahl 2006 zutreffen, bei der sich CDU und SPD angesichts ihres noch frischen Bündnisses auf Bundesebene mit gegenseitigen Attacken weitgehend zurückhielten. Wie weit trägt nun aber die Erklärung, dass bundespolitische Konstellationen das Landtagswahlverhalten in Rheinland-Pfalz beeinflusst haben?

\section{Der Einfluss der Bundespolitik auf Landtagswahlen aus Sicht der amtlichen Statistik}

Um die Frage nach dem bundespolitischen Einfluss mit Daten der amtlichen Statistik zu überprüfen, wurde von Reiner Dinkel folgendes Verfahren vorgeschlagen: Aus den Ergebnissen bei zwei aufeinander folgenden Bundestagswahlen und der zeitlichen Positionierung der dazwischen durchgeführten Landtagswahl im Bundestagswahlzyklus wird - unter der Annahme, dass sich der Stimmenanteil zwischen den nationalen Wahlen kontinuierlich entwickelt - für jede Partei der jeweils zu erwartende Stimmenanteil bei dieser Landtagswahl berechnet. ${ }^{23}$ Zum Beispiel fand die rheinland-pfälzische Landtagswahl 2001910 Tage nach der Bundestagswahl 1998 statt; 62,5 Prozent der insgesamt 1456 Tage dauernden Bundestagslegislaturperiode waren zu diesem Zeitpunkt also bereits verstrichen. Bei der

17 Peter Haungs / Eckhard Jesse, Die rheinland-pfälzische Landtagswahl vom 18. März 1979. Anfang vom Ende der CDU-Mehrheit?, in: ZParl, 11. Jg. (1980), H. 2, S. 154.

18 Peter Haungs / Eckhard Jesse, a.a.O. (1983).

19 Werner Billing, a.a.O., S. 585.

20 Angelika Scheuer, Die rheinland-pfälzische Landtagswahl vom 24. März 1996. SPD-FDP-Erfolg trotz struktureller Nachteile, in: ZParl, 27. Jg. (1996), H. 4, S. 617 f.

21 Cornelia Weins, Die rheinland-pfälzische Landtagswahl vom 25. März 2001. Klarer Sieg für Kurt Beck und die SPD, in: ZParl, 33. Jg. (2002), H. 1, S. 31.

22 Klaus G. Troitzsch, Die Wahlen zum Berliner Abgeordnetenhaus und zu den Landtagen von Rheinland-Pfalz und Schleswig-Holstein, in: ZParl, 2. Jg. (1971), H. 4, S. 434 ff.; Werner Billing, Die rheinland-pfälzische Landtagswahl vom 17. Mai 1987: CDU verliert alleinige Regierungsmacht - Koalition nach Bonner Muster, in: ZParl, 19. Jg. (1988), H. 1, S. 9.

23 Vgl. Reiner Dinkel, Der Zusammenhang zwischen Bundes- und Landtagswahlergebnissen, in: PVS, 18. Jg. (1977), S. 348 ff.; siehe ebenso ders., Zur Gesetzmäßigkeit der Trendverschiebungen zwischen Landtags- und Bundestagswahlen, in: ZParl, 12. Jg. (1981), H. 1, S. 135 ff. sowie ders., Landtagswahlen unter dem Einfluß der Bundespolitik: Die Erfahrungen der letzten Legislaturperioden, in: Jürgen W. Falter / Hans Rattinger / Klaus G. Troitzsch (Hrsg.), Wahlen und politische Einstellungen in der Bundesrepublik Deutschland. Neuere Entwicklungen der Forschung, Frankfurt am Main 1989, S. 253 - 262. Vgl. auch das Eingehen auf Dinkel im Beitrag von Kai-Uwe Schnapp in diesem Heft der ZParl. 
Bundestagswahl 1998 holte die CDU 39,1 Prozent, bei der Bundestagswahl 2002 40,2 Prozent. Wäre der Wählerzuspruch zwischen den beiden Wahlen gleichmäßig angewachsen, hätte die CDU zum Zeitpunkt der Landtagswahl von einem Stimmenanteil von 39,8 Prozent ausgehen können. Tatsächlich konnte sie aber nur 35,3 Prozent der Zweitstimmen auf sich vereinigen; relativ zu dem erwarteten Stimmenanteil sind das 88,7 Prozent. Allgemein kann man also sagen, dass ein relativer Stimmenanteil von weniger als 100 Prozent anzeigt, dass eine Partei bei einer Landtagswahl weniger erfolgreich war, als dies aufgrund der Bundestagswahlergebnisse zu erwarten gewesen wäre. Umgekehrt ist ein relativer Stimmenanteil größer als 100 Prozent ein Zeichen dafür, dass mit dem realisierten Landtagswahlergebnis die Erwartungen übertroffen wurden.

Es zeigt sich, dass der Wahlerfolg der verschiedenen Parteien in Rheinland-Pfalz zum Teil ganz erheblich von den Ergebnissen abweicht, die aufgrund der jeweiligen vorhergehenden beziehungsweise nachfolgenden Bundestagswahl zu erwarten gewesen wären (vgl. Tabelle 2). Gemäß der erläuterten Logik ist also bei jeder Wahl und praktisch jeder Partei ein Einfluss der Bundespolitik zu erkennen, der dazu geführt hat, dass erwartete und realisierte Stimmenanteile voneinander abweichen. CDU und FDP haben dabei in der Mehrzahl der Wahlen überdurchschnittlich schlecht abgeschnitten; ihr mittlerer relativer Stimmenanteil liegt bei 95,5 beziehungsweise 88,0 Prozent (vgl. die erste Zeile in Tabelle 3). SPD (Mittelwert: 106,5 Prozent), Bündnis 90/Die Grünen (103,5 Prozent) sowie insbesondere die sonstigen Parteien (137,4 Prozent) haben hingegen im Land in aller Regel bessere Ergebnisse erzielt als bei Bundestagswahlen. Da sich dieses Muster nicht von Wahl zu Wahl ändert, sondern längere Perioden günstiger und ungünstiger Landtagswahlergebnisse $\mathrm{zu}$ beobachten sind, ist davon auszugehen, dass die Bundespolitik einen systematischen Einfluss auf Landtagswahlen ausübt. ${ }^{24}$

\begin{tabular}{|c|c|c|c|c|c|}
\hline \multicolumn{6}{|c|}{$\begin{array}{l}\text { Tabelle 2: Relative Stimmenanteile bei Landtagswahlen in Rheinland-Pfalz, } 1951 \text { bis } 2001 \\
\text { (Angaben in Prozent) }\end{array}$} \\
\hline Jahr & $\mathrm{CDU}$ & SPD & FDP & B90/Grüne & Sonstige \\
\hline 1951 & 77,9 & 121,4 & 117,2 & - & 135,8 \\
\hline 1955 & 88,7 & 111,1 & 114,0 & - & 116,5 \\
\hline 1959 & 93,5 & 110,3 & 87,0 & - & 129,0 \\
\hline 1963 & 90,5 & 117,2 & 83,8 & - & 115,1 \\
\hline 1967 & 95,9 & 96,7 & 95,9 & - & 178,6 \\
\hline 1971 & 106,6 & 95,6 & 82,6 & - & 97,4 \\
\hline 1975 & 111,7 & 89,5 & 71,8 & - & 193,6 \\
\hline 1979 & 106,0 & 99,8 & 71,5 & - & 183,2 \\
\hline 1983 & 104,6 & 103,1 & 50,0 & 100,0 & 100,0 \\
\hline 1987 & 99,9 & 104,8 & 79,3 & 81,7 & 193,5 \\
\hline 1991 & 85,2 & 122,9 & 68,7 & 153,7 & 84,4 \\
\hline 1996 & 91,8 & 99,3 & 127,7 & 111,9 & 122,8 \\
\hline 2001 & 88,7 & 113,6 & 92,0 & 72,0 & 135,6 \\
\hline \multicolumn{6}{|c|}{$\begin{array}{l}\text { Die Berechnung erfolgte nach dem Verfahren von Reiner Dinkel. Für die Landtagswahlen } 1947 \text { und } \\
2006 \text { ist eine Berechnung relativer Stimmenanteile nicht möglich, da diese Wahlen nicht von einem } \\
\text { Bundestagswahlpaar „eingerahmt“ werden. } \\
\text { Quelle: Eigene Berechnungen. }\end{array}$} \\
\hline
\end{tabular}

24 Vgl. Reiner Dinkel, a.a.O. (1977), S. 349. 
Diese Systematik lässt sich besonders gut verdeutlichen, wenn man den relativen Stimmenanteil der Parteien für die Jahre, in denen sie am Vorabend einer Landtagswahl auch im Bund regierten, mit jenen Jahren vergleicht, in denen sie in Bonn beziehungsweise Berlin in der Opposition saßen (vgl. Tabelle 3). Die Beziehung ist eindeutig: CDU, SPD, FDP und Bündnis 90/Die Grünen haben bei rheinland-pfälzischen Landtagswahlen immer dann besonders schlecht abgeschnitten, wenn sie auch im Bund in der Regierungsverantwortung standen. Waren sie dort hingegen auf den Oppositionsbänken zu finden, fielen die Landtagswahlergebnisse überdurchschnittlich gut aus. Landtagswahlen sind aus dieser Perspektive also Wahlen, bei denen die im Bund regierenden Parteien abgestraft werden. ${ }^{25}$ Umgekehrt ist aber auch zu erkennen, dass die Bundespolitik keinesfalls die Wahlen zum Mainzer Landtag dominiert, denn auch die Leistungen der dort die Mehrheit haltenden Parteien haben einen systematischen Einfluss auf das Wahlergebnis: Immer dann, wenn CDU, SPD oder FDP die Landesregierung stellten, schnitten sie bei Landtagswahlen deutlich besser $\mathrm{ab}$, als wenn sie sich in Mainz in der Opposition befanden. Die Regierungsarbeit wurde also bei Landtagswahlen belohnt.

\begin{tabular}{|c|c|c|c|c|}
\hline \multicolumn{5}{|c|}{ 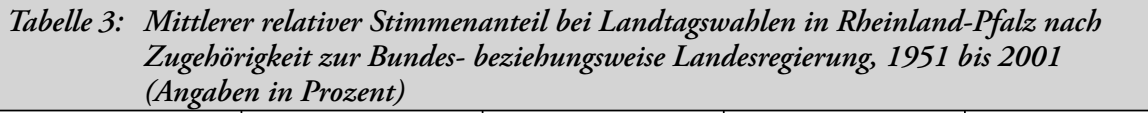 } \\
\hline & $\mathrm{CDU}$ & SPD & FDP & B90/Grüne \\
\hline Gesamt & $95,5(13)$ & $106,5(13)$ & $88,0(13)$ & $103,5(5)$ \\
\hline \multicolumn{5}{|c|}{ Bund } \\
\hline Regierung & $92,1(9)$ & $99,1(5)$ & $86,9(11)$ & $71,9(1)$ \\
\hline Opposition & $103,3(4)$ & $111,1(8)$ & $94,2(2)$ & $111,4(4)$ \\
\hline \multicolumn{5}{|c|}{ Land } \\
\hline Regierung & $96,5(11)$ & $111,4(3)$ & $94,3(8)$ & - \\
\hline Opposition & $90,2(2)$ & $105,0(10)$ & $78,1(5)$ & $103,5(5)$ \\
\hline
\end{tabular}

25 Dieser Zusammenhang fällt besonders stark aus, wenn Landtagswahlen in der Mitte der Bundestagslegislaturperiode abgehalten werden; vgl. zum Beispiel Reiner Dinkel, a.a.O. (1977), S. 351. In jüngster Zeit mehren sich jedoch die Hinweise, dass sich diese Beziehung auflöst; vgl. hierzu Simone Burkhart, Parteipolitikverflechtung. Über den Einfluss der Bundespolitik auf Landtagswahlentscheidungen von 1976 bis 2000, in: PVS, 36. Jg. (2005), S. 14 ff., Frank Decker / Julia von Blumenthal, Die bundespolitische Durchdringung der Landtagswahlen. Eine empirische Analyse von 1970 bis 2001, in: ZParl, 33. Jg. (2002), H. 1, S. 144 ff., Daniel Hough / Charlie Jeffery, Regionalwahlen in Mehr-Ebenen-Systemen, in: Thomas Conzelmann / Michèle Knodt (Hrsg.), Regionales Europa - Europäisierte Regionen, Frankfurt am Main 2002, S. 213 ff., Daniel Hough I Charlie Jeffrey, Landtagswahlen. Bundestestwahlen oder Regionalwahlen?, in: ZParl, 34. Jg. (2003), H. 1, S. 79 ff. Auch für Rheinland-Pfalz lässt sich dieser Zusammenhang nicht für alle Parteien nachweisen; vgl. Jürgen Maier, Aus Schwarz mach' rot: Der Einfluss der Bundespolitik auf das Stimmverhalten bei Landtagswahlen in Rheinland-Pfalz, in: Kerstin Völkl / Kai-Uwe Schnapp / Oscar W. Gabriel / Everhard Holtmann (Hrsg.), Wähler und Landtagswahlen in der Bundesrepublik Deutschland, Baden-Baden 2007 (i.E.). 
Berücksichtigt man einerseits, dass die Mitgliedschaft in Bundes- und Landesregierung bei den Parteien in verschiedenen Kombinationen auftritt und sich auf diese Weise bundesund landespolitische Effekte teilweise verstärken, teilweise aber auch aufheben, sowie andererseits, dass das Gewicht der bundespolitischen Einflüsse von der jeweiligen Positionierung der Landtagswahl im nationalen Wahlzyklus abhängt, ergibt sich ein geringfügig anderes Bild $^{26}$ : Multivariate Analysen zeigen, dass sich - mit Ausnahme von Bündnis 90/Die Grünen - eine Regierungsbeteiligung im Bund für alle Parteien negativ auswirkt. Umgekehrt ist die Mitgliedschaft in der Landesregierung nur für CDU und SPD von Vorteil, während die FDP hierdurch Stimmen einbüßt. Mit anderen Worten: CDU und SPD erzielen bei rheinland-pfälzischen Landtagswahlen die besten Ergebnisse, wenn sie im Bund in der Opposition sitzen, im Land hingegen regieren. Besonders schlecht schneiden die Parteien ab, die in der Bundeshauptstadt regieren, in der Landeshauptstadt hingegen opponieren. Die günstigste Konstellation für die FDP ergibt sich, wenn die Liberalen in Bonn beziehungsweise Berlin und in Mainz auf den Oppositionsbänken Platz genommen haben. Sind sie hingegen an beiden Standorten an den Hebeln der Macht zu finden, sind besonders schlechte Landtagswahlergebnisse die Folge. Allerdings ist vor allem für die beiden Volksparteien festzuhalten, dass der gemessene Effekt der Landespolitik deutlich geringer ausfällt als der Einfluss der Bundespolitik. Bundespolitischer Gegenwind lässt sich also nicht durch günstige landespolitische Vorzeichen kompensieren. Hingegen können strategische Nachteile im Land durch Rückenwind aus der Bundeshauptstadt mehr als wettgemacht werden.

\section{Der Einfluss der Bundespolitik auf Landtagswahlen aus Sicht von Wahlumfragen}

Weitere Hinweise auf die Bedeutung von bundespolitischen Einflüssen für das Landtagswahlverhalten der Rheinland-Pfälzer erhält man, wenn man sie danach befragt, ob die Politik im Bund oder die Politik im Land für ihre Wahlentscheidung von größerer Wichtigkeit ist. Die Ergebnisse legen nahe, dass empirische Analysen auf Basis amtlicher Daten die Bedeutung der Bundesebene überschätzen ${ }^{27}$ : So gaben zur Landtagswahl 1983, die nach Meinung politischer Beobachter erstens aufgrund des Zusammenbruchs der SPD/FDPKoalition in Bonn und zweitens durch die in Deutschland bis dahin einmalige Zusammenlegung von Bundes- und Landtagswahl unter extremem Einfluss der Bundespolitik stand, nur 51 Prozent an, dass die Bundespolitik wichtiger sei, 47 Prozent nannten die Landespolitik und ein Prozent bekannte, dass beide Ebenen gleich wichtig sind. 1991 - ebenfalls eine Wahl unter starkem Eindruck bundespolitischer Ereignisse - fanden 38 Prozent die Bundespolitik und 32 Prozent die Landespolitik bedeutsamer; 28 Prozent waren unentschieden. ${ }^{28}$ Bei der Landtagswahl 2001 - erneut eine Wahl mit eher bundespolitischem Charakter - orientierten sich 40 Prozent der Wähler an der Bundes-, aber 47 Prozent an der Landespolitik. 2006, als die Wahl mit vorrangig landespolitischen Themen bestritten

26 Vgl. Jürgen Maier, a.a.O.

27 Vgl. hierzu - soweit nicht anders ausgewiesen - die im Zentralarchiv für empirische Sozialforschung an der Universität zu Köln eingestellten Umfragedaten: ZA 1250 (1983), ZA 3382 (2001), ZA 4401 (2006).

28 Vgl. Werner Billing, a.a.O. (1991), S. 597. 
wurde, befanden 39 Prozent die Politik auf Bundesebene, 50 Prozent die Landespolitik für wichtiger. Insgesamt ist also kein eindeutiger Zusammenhang zwischen der Bedeutung, die politische Analysten bundespolitischen Einflüssen beimessen, und den Selbstauskünften von Wählern, an welcher Politikebene sie ihre Stimmenabgabe ausrichten, zu erkennen.

Im Normalfall sind solche Selbsteinschätzungen mit großer Vorsicht zu genießen, denn in Befragungen versuchen Wähler zum einen Antworten zu erteilen, die möglichst mit von ihnen antizipierten Mehrheitsmeinungen übereinstimmen; unabhängig davon nehmen sie zum anderen Politik häufig durch die parteipolitische Brille wahr - und verzerren damit objektiv vorhandene Sachverhalte so, dass sich diese möglichst widerstandslos in ihr eigenes, höchst subjektives Bild von der Welt einfügen. Im vorliegenden Fall jedoch bestätigen komplexere Analysen die von den Befragten vorgenommene Gleichgewichtung bundesund landespolitischer Einflussgrößen. ${ }^{29}$ Die auf der Basis multipler Regressionsanalysen geschätzten Erklärungsbeiträge der beiden Politikebenen fallen - alles in allem - sehr ähnlich aus. ${ }^{30}$

Ein systematisches Übergewicht der Bundes- oder der Landespolitik lässt sich weder für einzelne Wahlen noch für bestimmte Parteien erkennen. Ebenso wenig sind eindeutige Trends über mehrere Landtagswahlen hinweg zu beobachten. Bemerkenswert ist jedoch, dass Einstellungen zur Bundes- und zur Landespolitik (gemessen über die Bewertung der Regierungsleistungen beziehungsweise über die Sympathiebewertung des Regierungschefs) im Vergleich zur Parteibindung nur einen relativ geringen Erklärungsbeitrag leisten. Trotz der Abschleifung längerfristiger parteipolitischer Loyalitäten gilt also - analog zu anderen Bundesländern ${ }^{31}$ - auch in Rheinland-Pfalz, dass die Parteiidentifikation nach wie vor der bedeutendste Bestimmungsfaktor der individuellen Wahlentscheidung ist.

\section{Zusammenfassung}

(1) Die politische Landschaft in Rheinland-Pfalz hat sich in den letzten zwei Jahrzehnten dramatisch verändert. Aus einem einstigen Stammland der CDU ist eine Bastion der Sozialdemokratie geworden.

(2) Ursache für diese Entwicklung ist die Auflösung tradierter Bindungen der katholischen Bevölkerungsmehrheit an die CDU. Die Erosion der Parteiloyalitäten in dieser Wählergruppe lässt sich auf allgemeine Modernisierungs- und Säkularisierungsprozesse sowie auf eine Reihe hausgemachter Fehlleistungen der CDU zurückführen.

(3) Aufgrund der Flexibilisierung des Wahlverhaltens ist zu erwarten, dass die Bedeutung sozialstrukturell bedingter, längerfristiger Bindungen an politische Parteien für das indi-

$29 \mathrm{Vgl}$. hierzu ausführlich Jürgen Maier, a.a.O.

$30 \mathrm{Zu}$ diesem Ergebnis kommt auch Daniela Klos, Motivtransfer bei Nebenwahlen: Ein Vergleich wahlspezifischer und bundespolitischer Einflussfaktoren auf die Wahlentscheidung bei der hessischen Landtagswahl und der Europawahl in Deutschland 1999, in: Frank Brettschneider / Jan W. van Deth / Edeltraud Roller (Hrsg.), Europäische Integration in der öffentlichen Meinung, Opladen 2003, S. 335 ff., die als erste und bisher einzige empirisch den Einfluss von Bundes- und Landespolitik auf der Individualebene untersucht hat.

31 Vgl. Oscar W. Gabriel, Die baden-württembergische Landtagswahl vom 25. März 2001. Fehlschlag einer „Teufelsaustreibung“, in: ZParl, 33. Jg. (2002), H. 1, S. 10 ff.; Daniela Klos, a.a.O., S. $345 \mathrm{ff}$. 
viduelle Stimmverhalten gesunken ist. Im Gegenzug sollten kurzfristige politische Orientierungen - unter anderem auch der Einfluss bundespolitischer Konstellationen - an Einfluss gewonnen haben.

(4) Auf der Aggregatebene lassen sich deutliche Einflüsse der Bundes- und der Landespolitik auf das Abschneiden von CDU, SPD, FDP, Bündnis 90/Die Grünen sowie der sonstigen Parteien bei rheinland-pfälzischen Landtagswahlen erkennen. Parteien, die im Bund regieren, schneiden bei Landtagswahlen systematisch schlechter ab als Parteien, die in Bonn beziehungsweise Berlin die Opposition stellen. Mit Ausnahme der FDP führt eine Regierungsbeteiligung im Land hingegen zu überdurchschnittlich guten Wahlergebnissen. Im Allgemeinen fällt jedoch das Gewicht bundespolitischer Faktoren größer aus als der Einfluss landespolitischer Konstellationen.

(5) Auf der Individualebene ist dieses Muster nicht zu erkennen. Zum einen ist die Bedeutung von Bundes- und Landespolitik für das Landtagswahlverhalten der RheinlandPfälzer gering; die Stimmenabgabe wird nach wie vor vor allem von der Parteibindung beeinflusst. Zum anderen ist weder ein generelles Übergewicht bundespolitischer noch eine systematisch größere Bedeutung landespolitischer Faktoren zu erkennen. Dies gilt über alle untersuchten rheinland-pfälzischen Landtagswahlen und Parteien hinweg.

\section{Landtagswahlen in Bayern 1966 bis 2003: verstärkte bundespolitische Durchdringung aufgrund der Doppelrolle der CSU}

\section{Harald Schoen}

Den Bayern ist es besser als den Bürgern anderer Bundesländer gelungen, die Aufmerksamkeit der häufig am Verhalten des deutschen Durchschnittswählers interessierten Wahlforschung auf sich zu ziehen. Eindrücklichstes Beispiel dafür ist die Kontroverse zwischen Jürgen W. Falter und Alf Mintzel in dieser Zeitschrift über die Besonderheiten der bayerischen Wähler. ${ }^{1}$ Obgleich darin spezifisch bayerisches Wahlverhalten vermutet wurde, ging die Forschung nicht so weit anzunehmen, Bayerns Bürger würden die Bundespolitik vollkommen außer Acht lassen, wenn sie über die Zusammensetzung des Landtages entscheiden, würden also in diesem Sinn unverwechselbar bayerisch wählen. Im Gegenteil, zahlreiche Analysen gehen ausdrücklich von bundespolitischen Einflüssen auf bayerisches Landtagswahlverhalten aus. So habe die CSU in den siebziger Jahren „uneingeschränkt von einem aus der Dialektik zwischen Bundes- und Landtagswahlen folgenden, Oppositionsef-

1 Siehe Jürgen W. Falter, Bayerns Uhren gehen wirklich anders. Politische Verhaltens- und Einstellungsunterschiede zwischen Bayern und dem Rest der Republik, in: ZParl, 13. Jg. (1982), H. 4, S. 504 - 521; Alf Mintzel, Gehen Bayerns Uhren wirklich anders?, in: ZParl, 18. Jg. (1987), H. 1, S. 77 - 93; Jürgen W. Falter, Wie gehen sie denn nun wirklich, die bayerischen Uhren?, in: ZParl, 19. Jg. (1988), H. 1, S. 113 f. 